INTERNATIONAL DESIGN CONFERENCE - DESIGN 2018

https://doi.org/10.21278/idc.2018.0234

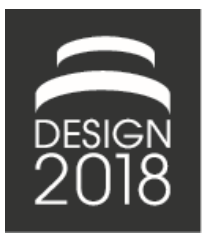

\title{
SCALING OF STRUCTURAL COMPONENTS BY KNOWLEDGE-BASED ENGINEERING METHODS
}

\author{
P. Wolniak, B. Sauthoff and R. Lachmayer
}

\begin{abstract}
Decreasing the development time is a significant mean for a company operating in the technical area to lower the development cost. Effort invested into developing a given product can be used to derive following variants of the same product by applying a scaling method. Common scaling methods are created in a means of a geometrical scaling without the option of a flexible change of geometry, while maintaining the concept in the main. Therefore a method is proposed for deriving a knowledge based engineering system which ensures the beforehand mentioned characteristics of the scaling process.
\end{abstract}

Keywords: scaling, knowledge based engineering (KBE), structural component, design methods, numerical methods

\section{Introduction}

Companies that operate and produce products in the technical area are exposed to increasingly challenging market situations. The developed products should be individualized to the customers' needs while offering high quality at an acceptable price. The temporal and especially economic claims are constantly growing, forcing the companies to develop a given product that matches the cost-side as well as the technical requirements in a short period of time (Pahl et al., 2007).

Following an initial development, it is often necessary to provide further product variants regarding a modified geometry or performance. To reduce a following time-consuming development of those variants, it is possible to use the already designed "master-size" to derive other possible modifications. By using this kind of scaling process, it is possible to divide it into two areas of application (Pahl et al., 2007):

- Series development with defined increments

- Mass customization for customer-specific changes

This paper proposes a method for building a knowledge-based engineering system with the explicit objective of a geometrical scaling purpose. The main focus is on products from the oil and gas drilling industry, where the exploration of hydrocarbons is executed by using kilometre-long drilling strings under extreme conditions. In this industry the development of drilling tools typically starts with a small size tool and is then transferred to consecutives sizes. But also other industries with the need for consecutive developments starting from an initial development can use the proposed approach. A possible industry in this context could be the aviation industry, where structural components like ribs or stringers have a big significance and where often product families of airplanes have a similar composition. For this purpose, a hybrid system between the aforementioned areas of scaling applications is developed. The objective is to use defined increments of parameters that are typical in the oil and gas drilling industry as a framework while giving the developer the flexibility of an individual adaption of 
geometry or performance capacities related to the given project. The focus is on structural components in the industrial context of the drilling industry. That is, because similar structural components are repeatedly used throughout different and newly developed drilling tools. While these components often remain very similar on a conceptual side, an adaption to the given problem or new development is still needed, resulting in an increased development time.

Within the definition of a structural component as a function of a technical system, its main task is to absorb and transmit mechanical energy (Roth, 2000). In the context of the drilling industry this mainly represents drill strings that are directly exposed to environmental influences. These structural components must survive high temperature, pressure, bending or shocks and maintain their own functionality and the functionality of surrounding components (Lyons, 2005).

A special characteristic of structural components in this industrial context are different domains and their associated targets. Thus, often an additional optimization problem arises from a given scaling problem. The domains to be considered include, for example, the mechanical structure, ensuring the function or the cost. Objectives in a physical consideration may be the maximum strength, the maximum heat flow, or the maximum fluid flow velocity through an internal bore, which also may lead to tradeoffs. It is important to recognize and to create a relative weighting of these trade-offs to ensure the identification of an optimum and to integrate it into the process of scaling (Deimel, 2007).

\section{State of the art}

\subsection{Scaling in literature}

A geometrical example of the definition of similarity provides the consideration of two triangles that are regarded as similar if their side length ratios are constant. Such a ratio represents a simple form of a similarity term, which means that by maintaining this ratio the sizes are transferred geometrically similar (Stichlmair, 1990). Here, the ratio of two lengths represents an invariant about the length. More of these invariants are defined based on the SI system of units, out of which all similarities can be described from a dimensional point of view (Roth, 2000).

This consideration is taken up by the Buckingham Pi-theorem, which states that for every fully dimensional homogeneous relationship a dimensionless potency products relationship can be found (Bridgman, 1932). The result of this so called "Dimensional Analysis" is a group of dimensionless products that are valid for the problem on hand. The dimensional analysis can be stated from a relevance list where all important variables are listed in. If all determined dimensionless potency products are respected, the considered problem is fully transferable from, for example, a model to a real-size description. The disadvantage with this procedure is the very intuitive approach and the necessary deep understanding of the overall physical problem that may lead to an underestimation and a false determination of the dimensionless potency products. In addition, there are no direct analytical connections between those products.

Kline (1965) has shown extensions for the estimation of dimensionless similarity terms. He uses the "method of similitude" where an intuitive dimensionless force or energy condition is set up and through a balanced analytical equation dimensional groups can be determined. Other authors have taken up the approach of the dimensional analysis, as the method of determining similarity terms on the basis of the Pi-theorem, and expanded it (Görtler, 1975; Baker et al., 1991)

The formulation of the series development as stated by Pahl et al. (2007) rather focuses on the product development. In this connection the previously mentioned dimensional invariants are used as a means of a complete similarity or a half-similarity. The definition of the changing increments is based on the decimal geometric series, and thus sets a standard for the development and production. The used relationships are of rather simple nature and mainly describe a geometrical similarity or the transfer of performance and other component-specific terms. The scaling is done without a possible conceptual change, so that design features and layout remain the same.

Rudolph (2002), however, uses the similarity terms and applies his approaches on different problems. In this, he mainly uses the parameter reduction and associated simplification of equations and contexts that result from the dimensional analysis. For example, he presents a way to build neural networks by using similarity. Further, part of his work focuses on the development of a design language, as well as 
the distinction between a description and an evaluation of design solutions in a dimensionless mathematical space.

Another approach is presented by Deimel and Franke (2006) and by Deimel (2007) in their works on the integration of similarity terms into the development process. By using a so-called "task identification," they are able to describe individual points of the design and use this description in a means of a comparison. As a result of a dimensionless consideration of the leading analytical equations, sensitivities can be found, which can then be used to perform an optimization. Koschorrek (2007) refers in his work more on the conceptual phase of the design process. In this, he also uses a dimensionless consideration for a description and functional evaluation of individual solutions.

A model-based modification and optimization of components is supported by a sizing-optimization. In this method a parametric CAD model is optimized by defining a solution space in regard to a target size and the modifiable parameters. An optimization algorithm then varies the parameters in a defined range regarding a given target function. Based on the solution of a finite element software, the topology is modified and adapted regarding stresses for the investigated case of loading (Saitou, 2005).

Another way of scaling is proposed by Dutson and Wood (2002). The main idea is a decoupling of the geometric change of a component and the material behaviour in the scaling process. Especially with large differences between the geometric size of the source and the target model, the scaling may cause fluctuations in relation to the properties because of, for example, material inhomogeneities. Transformation matrices are used to merge the empirically determined data of the geometrical and material changes that lead to an overall improvement of the estimation quality.

\subsection{Alternative approach to methods known from literature}

From a detailed study of the presented methods, it becomes clear that the use of similarity methods leads to possibly good results, but this technique is subject to strong restrictions or the use and knowledge of explicit mathematical equations. For example, it is possible to perform a purely geometric scaling in terms of the presented series development (Pahl et al., 2007) and by this create new size variations. The description of the system properties and the derivation of corresponding similarity terms are confined only to selected mechanical and thermo-dynamical or manufacturing properties (Moog, 1985). The degrees of freedom for a change of design features or a conceptual change of the design are strongly limited. An optimization or improved utilization of existing properties becomes almost impossible.

Although dimensional analysis models the physics of a problem and therefore offers many possibilities to scale and solve a physical problem, it does not help in changing a complex geometry of a structural component.

Regarding the further introduced methods, no primary focus on the flexible change of geometrical properties or topology is identifiable. Using the approaches of Deimel and Franke (2006) and Deimel (2007) optimizations within the scaling process, especially relative to possible trade-offs, are observable, describable and applicable. Still, a comprehensive process is not given for a scaling and simultaneous use of the trade-off analysis relative to a following configuration of the component. The very broad results of Rudolph (2002) in the area of similarity terms are usable in many areas of development. However, a direct connection from a master size to a following variant design is not a main objective of the work.

Table 1 gives an additional overview of the considered methods and their applicability to a specific issue. A high diversity and complexity in regards to the methods is recognizable, which means that different methods have a different applicability, depending on the considered problem.

The advantages of the sizing optimization in terms of numerical modeling are invalidated by the disadvantage of the occurring model instability regarding changes of the topology and the related high manual preparation. In addition, the transmission of the shape modified by the FE program is only possible semi-automatically, which implicates a temporal disadvantage. Besides, only mechanical target figures are optimizable (Saitou, 2005).

The empirical similitude method possesses a higher quality of results towards the traditional scaling methods (dimensional analysis, series development). However, a significant effort is required to generate the results due to the necessary empirical data acquisition. If a geometric alteration is to be 
performed, the data acquisition must be repeated. This repeated acquisition increases the temporal effort and renders the application useless, especially at a conceptual phase (Dutson and Wood, 2002).

Table 1. Overview of presented methods from state of the art

\begin{tabular}{|c|c|c|c|c|}
\hline Method & $\begin{array}{c}\text { Geometric } \\
\text { dependencies }\end{array}$ & $\begin{array}{l}\text { Conceptual } \\
\text { changes }\end{array}$ & $\begin{array}{l}\text { Analytical and } \\
\text { mathematical } \\
\text { consideration }\end{array}$ & $\begin{array}{c}\text { Numerical } \\
\text { modeling }\end{array}$ \\
\hline Series development & Primary purpose & Not provided & $\begin{array}{c}\text { For simple } \\
\text { dependencies }\end{array}$ & Not provided \\
\hline $\begin{array}{c}\text { Similarity terms / } \\
\text { dimensional analysis }\end{array}$ & \multirow{3}{*}{$\begin{array}{l}\text { For simple } \\
\text { geometries }\end{array}$} & $\begin{array}{l}\text { Possible } \\
\text { derivation }\end{array}$ & $\begin{array}{c}\text { Suitable for static } \\
\text { and dynamic } \\
\text { problems }\end{array}$ & Not provided \\
\hline $\begin{array}{c}\text { Similarity terms (Deimel, } \\
2007)\end{array}$ & & Primary purpose & Medium & Medium \\
\hline $\begin{array}{c}\text { Similarity terms } \\
\text { (Koschorrek, 2007) }\end{array}$ & & Primary purpose & Medium & Medium \\
\hline $\begin{array}{l}\text { Case-based reasoning } \\
\quad \text { (Rudolph, 2002) }\end{array}$ & Independent & $\begin{array}{c}\text { Only with } \\
\text { existing concepts }\end{array}$ & $\begin{array}{c}\text { Only as a } \\
\text { derivation of } \\
\text { existing concepts }\end{array}$ & Not provided \\
\hline Sizing optimization & $\begin{array}{c}\text { As far as } \\
\text { supported by } \\
\text { CAD model }\end{array}$ & Not provided & $\begin{array}{c}\text { As far as } \\
\text { supported by FE- } \\
\text { program }\end{array}$ & $\begin{array}{c}\text { Only method } \\
\text { able to represent } \\
\text { mechanical field } \\
\text { problems }\end{array}$ \\
\hline $\begin{array}{c}\text { Empirical Similitude } \\
\text { Method }\end{array}$ & Very good & $\begin{array}{c}\text { Can be } \\
\text { empirically } \\
\text { assigned }\end{array}$ & $\begin{array}{c}\text { Through } \\
\text { transformational } \\
\text { matrices }\end{array}$ & Not provided \\
\hline
\end{tabular}

On the basis of the methods known from literature, no direct method is indicated for structural components and a subsequent scaling with simultaneous configuration. As previously described, this represents the main requirement for the generation of this method because it enables the scaling and adaption of frequently used components to the particular situation. Consequently, either strongly limited degrees of freedom exist for possible changes, or the change of a quantity implies a significant increase of the development time (empirical similitude method, sizing optimization). Therefore, a method is required that combines existing knowledge of the design process of a structural component and enables the interactive adaption and application of this knowledge. The established method for collecting, formalizing, and applying knowledge is knowledge-based engineering (KBE) (Koller, 1991). According to LaRocca (2012), the use of KBE methods has two basic objectives:

- Automation of repetitive and non-creative design tasks

- Support of multidisciplinary design optimization in all the phases of the design process

Therefore, the development of variant designs based on a master size can be interpreted as an iterative and automated development task. The second point of multidisciplinary optimization tasks follows on the occurring cross-domain target conflicts while scaling, which must be detected and resolved. Because of the applicability and the advantages of a KBE system, the scaling system is to be build using this form of knowledge representation.

In the following application, a KBE system can be developed as rule-based, case-based, or model-based. While a rule-based survey of knowledge follows on simple if/then relations, the case-based reasoning requires previously created case bases that are related to the particular problem by statistical methods. At the model-based reasoning, a model exists that recreates the configuration automatically and, as a consequence, accesses the knowledge. When creating a KBE system, the central question resides in the knowledge acquisition and the following compilation of the leading parameters for a subsequent application. The main assignment lies in the formalization of the knowledge that is available and implicitly applied by experts. To consider all possible aspects and to extract the knowledge from all possible sectors, it seems to be given to use a methodical approach for creating a KBE system. 
A methodical approach for creating a knowledge-based engineering system, methods such as MOKA or CommonKADS exists. In these methods an entire cycle is described that represents the formulation of a knowledge-based tool from the identification of the necessary knowledge through the implementation of formalized knowledge (Stokes, 2001). Sequentially, the required steps for scaling a structural component within the MOKA method are presented. For that, the MOKA life cycle is used, which consists of six steps.

\section{Methodical approach}

The general methodological approach of MOKA can be concretized for a KBE system based on the scaling of structural components derived from the presented steps. The entire MOKA method is a strongly iterative procedure. Between each point in the life cycle, a repeated change is possible and often necessary. In addition, the application should have a modular design so the individual points of interest can be processed independently. Figure 1 gives an overview of the general life-cycle steps and concrete sub-tasks. In the identify step of the life cycle, an examination is performed of the future use case of the KBE-system as well as the distinction of the implementation and the geometrical areas to be considered. The main focus of this paper is on the first four steps of the life cycle because of their crucial impact on the KBE system.

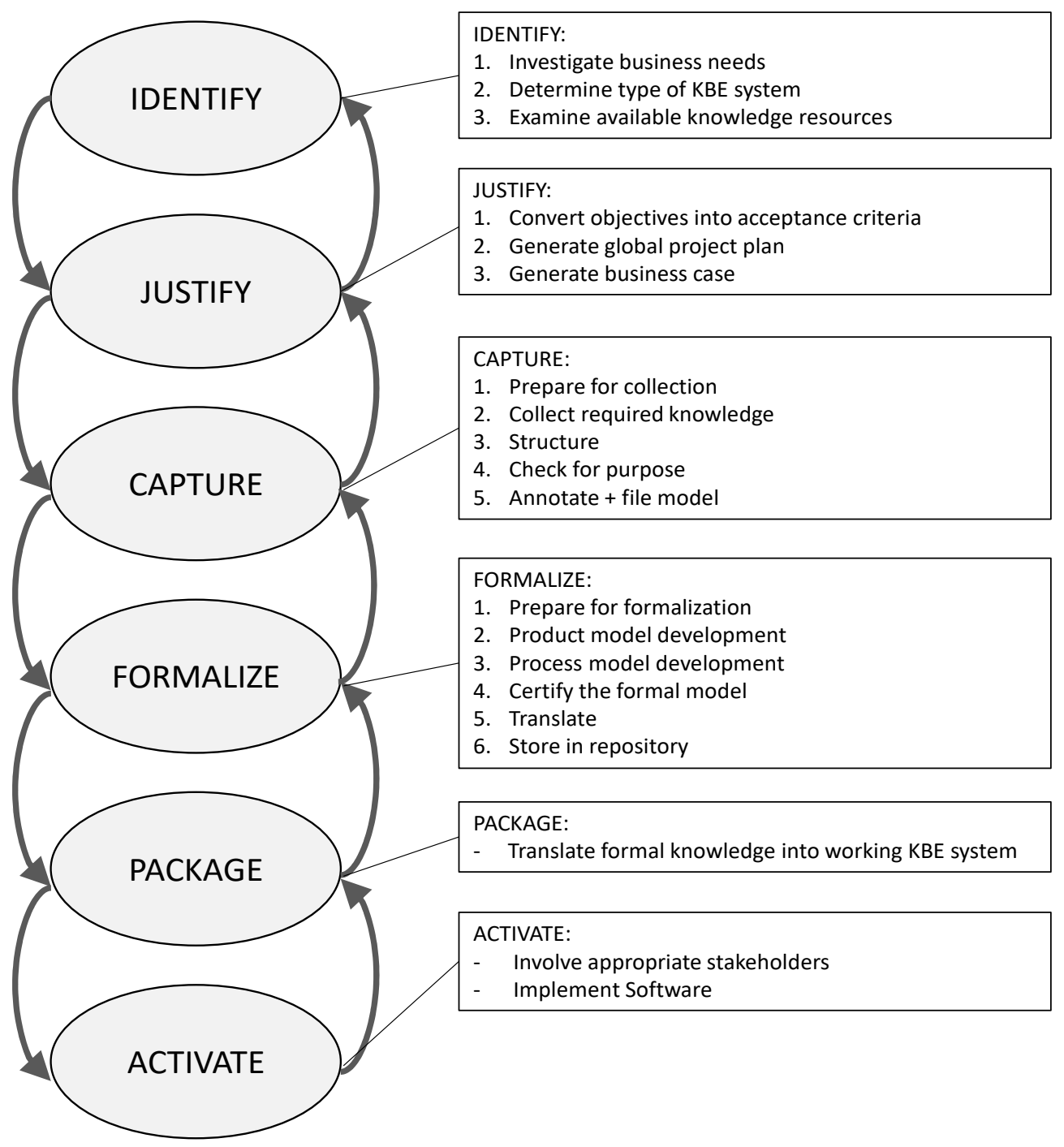

Figure 1. MOKA lifecycle steps (Stokes, 2001) 
"Justify" describes the creation of a project and a risk plan that stands for a justification of the proposed steps on a global level. The following steps "Capture" and "Formalize" represent a first rough structuring and a succeeding collection of necessary knowledge as well as the subsequent formalization as a preliminary step to the actual knowledge use. The "package" step represents a conversion of the formalized knowledge into a platform-specific software tool and an automation of the KBE system. As a last step, the activation of the KBE tool with the involvement of the appropriate stakeholders and the implementation in the company ends the MOKA lifecycle. Overall, the "Identify" and "Justify" steps are processed on a global level, while "Capture" and "Formalize" refer to a more local level with tangible component areas and references. The iterative cycle is especially used within the interaction of the knowledge capture and formalization, in exchange with the global identification. For example, while in the capture point it is possible to establish whether a certain component area should be considered more detailed through a loop back to the identify point. Linking this to the "Justify" step gives the possibility to examine the impact of a model simplification and to analyse it by adjusting the project and risk plan.

\section{Methodical approach transferred to structural component scaling}

Especially in the first two steps, general issues are edited and structured for the following knowledge capture. This includes considerations such as:

- Which boundary conditions change, and in which proportion?

- Is there a linear scaling of the loads?

- Does the component geometric change execute proportionally to the load change?

- Which areas of the component will be scaled, and which areas remain untouched?

- How do the interfaces to surrounding components change?

- How well is the design process documented?

- Who is in charge regarding design or requirement changes?

- What is the target platform?

These and other significant questions must be clarified and documented at first to ensure that all important aspects regarding global requirements are covered. The following project plan includes also a consideration of the KBE architecture and the amount of time and money to be invested.

The knowledge acquisition starts within the "Capture" step by identifying especially defined information sources, as well as the correct contact persons and experts on specific issues in a company. In addition, a standard for the documentation of extracted knowledge must be defined to ensure a subsequent formalization and later use of the knowledge.

The point of knowledge structuring requires a closer consideration of structural components. Consequently, a first step is to analyse the features of the component geometry and the outer shape. The term features in this case refers to geometric forms such as the shaping of various component areas, as well as functional distinctions such as different attachment concepts that also lead to different geometrical shaping, for example. All the geometrical and topological parameters are thereby captured and structured according to their importance regarding the possibility of a configurational change.

Depending on the problem, certain parameters cannot or should not be changed. This consideration is done on the basis of the specifications documented and changes permitted in the identify step. Moreover, the acquisition of the most important physical domains is effected parallel to the analysis of the design and calculation specification derived, for example, from past developments or practical experience from experts. The described domains include mechanics, thermodynamics or fluid dynamics. After the determination of individual scalable features, the associated main parameters must be concluded. This task can also be done by analysing similar components or past developments and projects as a reference point. Here, it is also possible to return to the first steps of the MOKA lifecycle and analyse the features and shape parameters one more time. 


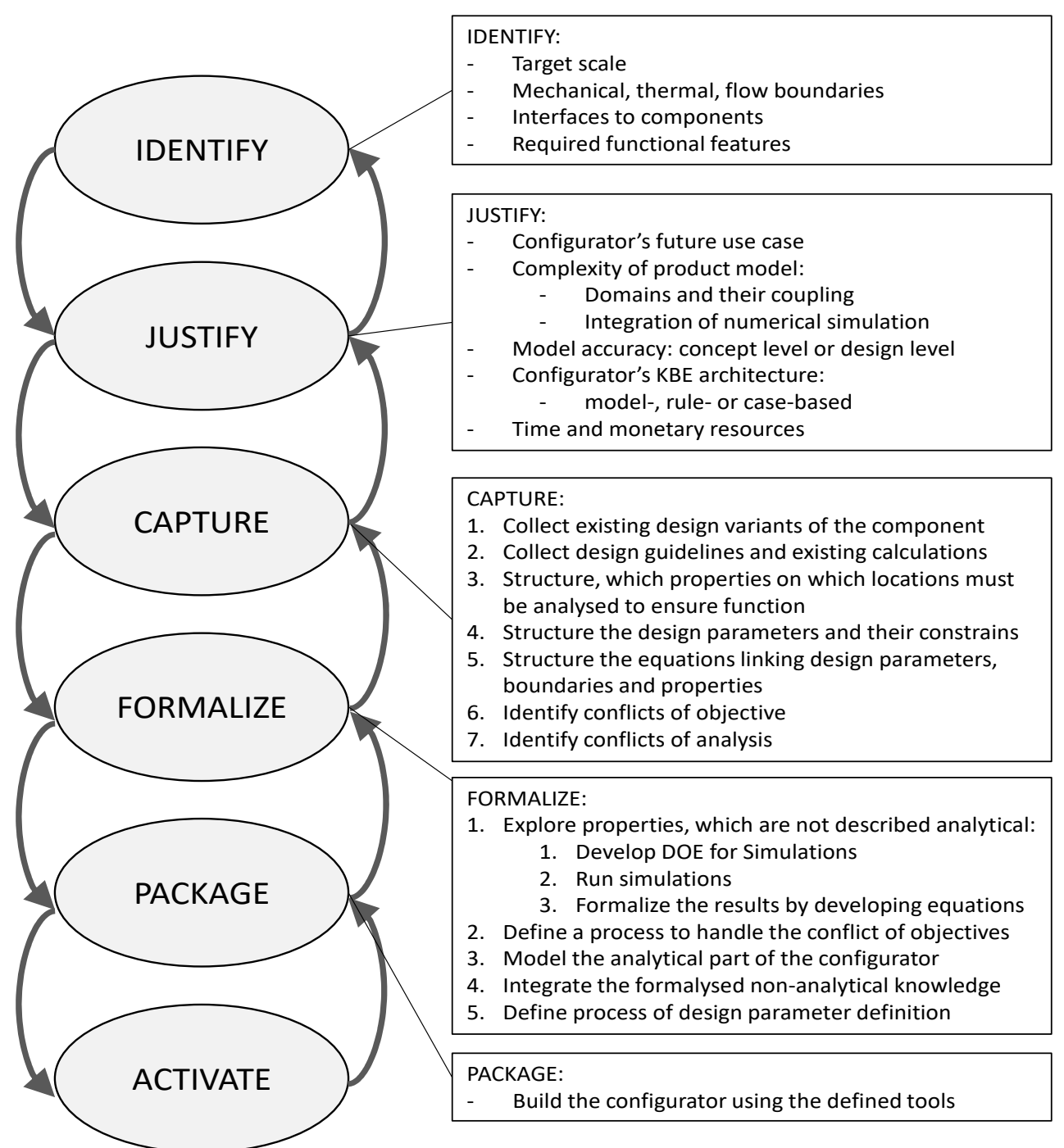

Figure 2. Adapted MOKA lifecycle to scaling of structural components

After finishing these steps, a delimitation of the design space is necessary to determine the scope of possible parameter changes. This delimitation prevents excessive complexity and a geometrically unsolvable constellation. Thereafter follows the determination of the necessary calculation steps for a development of the components according to the design process. By this design process it is possible to identify which geometrical areas can be described fully analytical and which areas must be considered with another analysis method. Therefore, the so-called "Entities" group from the ICARE forms, proposed by the MOKA method, can be abstracted and used for this purpose. For that, the component is divided into different areas, which then can be analysed for their degree of complexity and the extent to which it is useful to describe the scope of the component by using an analytical solution. At this point another iteration loop to the identify step is possible, where it can be determined how a particular feature that increases the complexity of the respective entity should be included in the formalization of knowledge. It is also possible to use a simplified model and exclude this feature at first for a later determination. After accomplishing these steps a first product model is created, by which it is possible to describe the structure, function, shape and the overall technology.

According to the classification of each component area and the affiliated calculation options, a KBE architecture can be created. For example, a constraint network can be set up precisely to limit the variance of the parameter scope. This creates a network of dependencies that are testable and applicable on a rule-based reasoning. In addition to the geometrical and topological solution space determination 
the variance of the external conditions, like for example the acting forces, the applied pressure or the interface to adjoining components must be taken into account for a correct component design.

After concluding all these entities the "Activity" group from the ICARE-forms can be used as a flow chart to represent all necessary steps for the design process. In this connection the initially established physical domains are used and based on that the necessary calculation and design steps can be determined. The rule-based concluding serves as a superordinate instance and reflects the whole design process. As intended in the MOKA method, the "Activity" group, and thereby the design process, can be divided into different parts that can relate, for example, to the provision of a certain type of stress, when relating to a mechanical domain, or overall to a specific area of the component. By that the socalled design process model is created that describes the external conditions and the design process. A clear instruction for designing a structural component is put forward by linking the product model and the design process model, as shown in Figure 3.

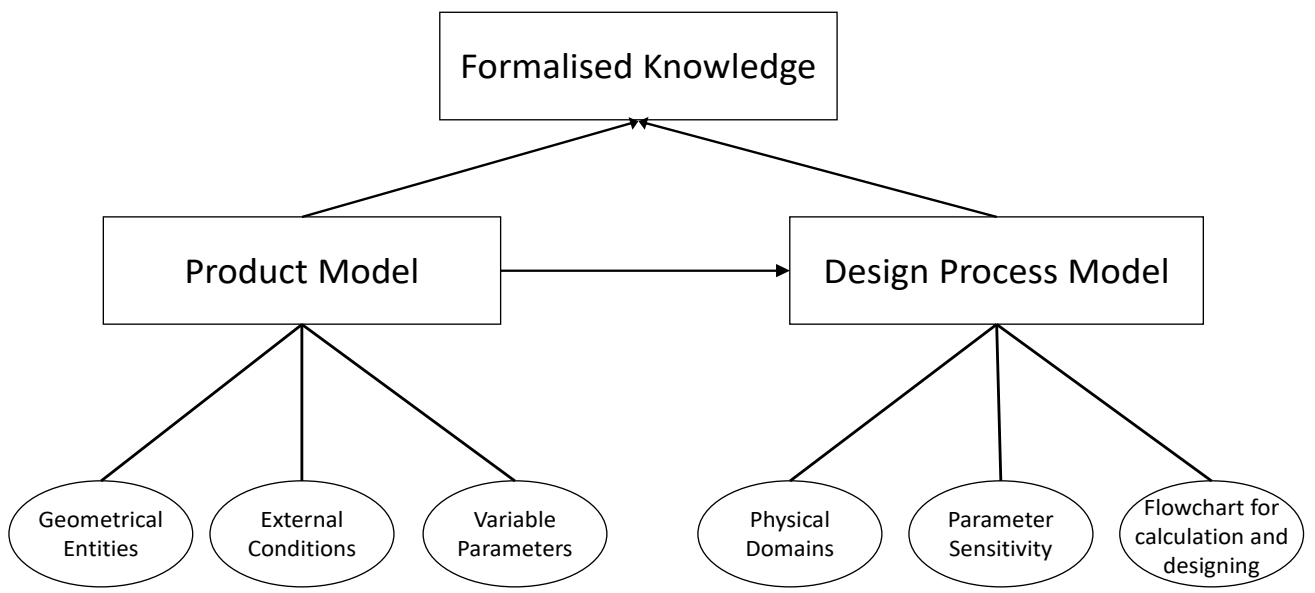

Figure 3. Knowledge formalised by linking product and design process model

Following the definition of an analysis method, analytically indescribable areas of the component are analysed by using, for example, the finite element method as a possible simulation method. For this analysis the areas classified as complex in the precedent "Entities" group are analysed for their leading parameters. It is also important to ascertain which dependencies prevail between those parameters. A combination of parameters is helpful, because it is possible to lower the amount of dependencies and simplify the later evaluation and formalization. For example, industry-specific increments can be exploited and the variance be set to be around those increments in a small margin. After establishing the most important parameters, a test plan according to, for example, the so-called "Design of Experiment" (DoE) method, can be made. According to this method, each parameter is changed gradually, and the impact on the overall system is documented for a parameter, such as mechanical stresses. The method is repeated for each remaining parameter. This method helps in the later formalization to create a KBE system that can create an output from these derived case-bases by a following approximation between the grid points determined in the FE-simulations.

At this point it is possible, by using the already provided analytical equations and the general relations as well as the main physical domains, to determine resulting trade-offs in the scaling process. From the mentioned analytical equations a parameter sensitivity is derivable, by using a dimensionless representation received through the combination with task-invariant parameters (Deimel, 2007). The impact of each parameter resulting from a change of the given parameter on each equation and domain is inferred. The information this sensitivity creates can be incorporated directly in the design process model, which leads to a kind of constraint by which iteratively an optimized solution for a specific problem can be found.

Next, the formalizing step is taken into account. The previously created analytical calculations, as well as the obtained results from the FE-simulations, must be prepared for a further use in the KBE system. In terms of the KBE architecture, this corresponds to the use of case-bases derived from specific problems. Based on the gradually changing parameters in the simulations, the results can be presented in a graph form. Statistical methods, such as the formation of the correlation coefficient and the following 
approximation of the gradient of the mechanical stress values based on the considered parameters, result in an approximated solution for the beforehand appointed analytically indescribable component areas.

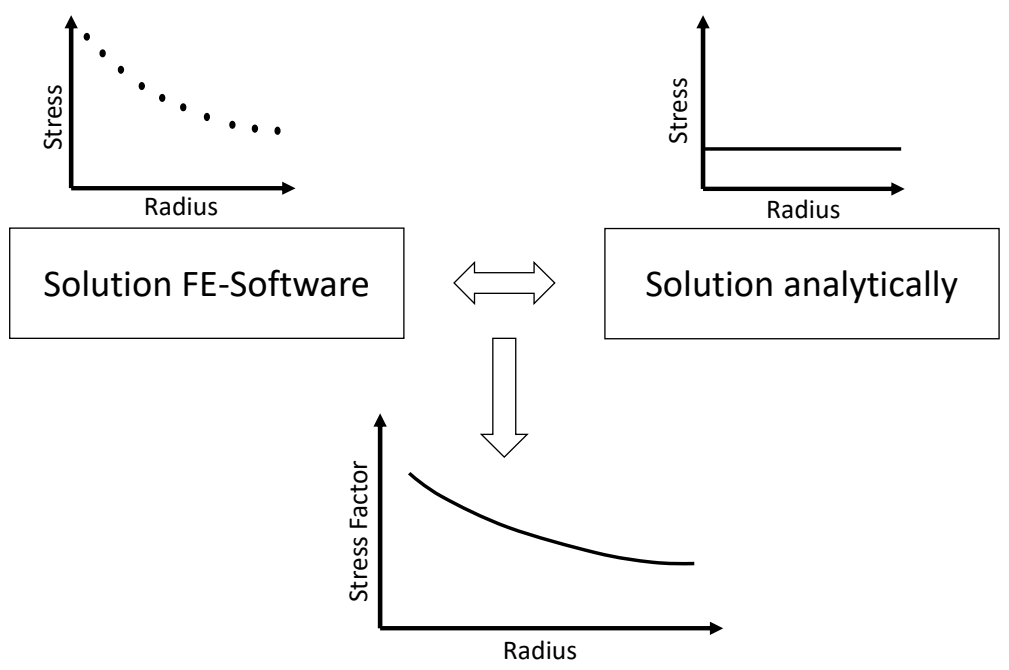

Figure 4. Combining case-based and analytical solutions for a formalized determination of analytically indescribable component areas

Furthermore, the approximated values can be used as absolute values derived directly from the casebases or as a relative factor while including the analytical calculations. For example, the evaluation of stresses in a notch area can be considered. For a simple case of a smooth cross-section, the stresses can be determined by an analytical means. The stress rise can be obtained by multiplying a certain factor depending on the notch shape by the analytical calculation at that point of interest. That certain factor is determined through the previously described case-bases. As shown in Figure 4, by using the analytical solution and the case-bases from the FE-software, it is possible to obtain the function of a stress factor at a given moment. By producing these functions at defined parameter sets, it is then possible to approximate a solution for the whole solution space.

\section{Case study}

Next, a case study to a selected component in the industrial context of the drilling industry is presented. The investigated component is a part of a drilling tool inside a drillstring, and its primary objective as a structural component is to accommodate electronical parts and assemblies. These electronics are mounted inside axially milled pockets that are distributed evenly over the circumference of the component, as seen in Figure 5.

As a means of protection against external influences, such as the aggressive drilling mud or the high hydrostatic pressure, a tubular cover is placed around the area with the mounted electronics and is preloaded by two shoulders and a thread, ensuring sealing and protection for the electronics.

A configurator for a size-scaling process is created and implemented into the spreadsheet software Microsoft Excel, with the specific target of an easy scaling, especially in the conceptual phase of the development, while still having the flexibility to configure an optimized outcome. By using the aforementioned method, a KBE system for the scaling of structural components based on MOKA is conceived. Due to the company's in-house experience in regard to the development of the component under consideration the steps identify and justify are accomplished by the help from subject matter experts and an additional use of a guideline for the design and calculation of this particular component. The previously described challenges like the estimation of the external conditions or the requirements in the scaling process are mostly answered by this guideline. Furthermore, simplifying assumptions are used, like for example the fixed outside diameter sizes that lead to a decrease of variables.

Alongside the known and internally documented external conditions the geometry of the component is examined for possible changes and a verification of the applicable complexity is determined. A first simplified model is created. 


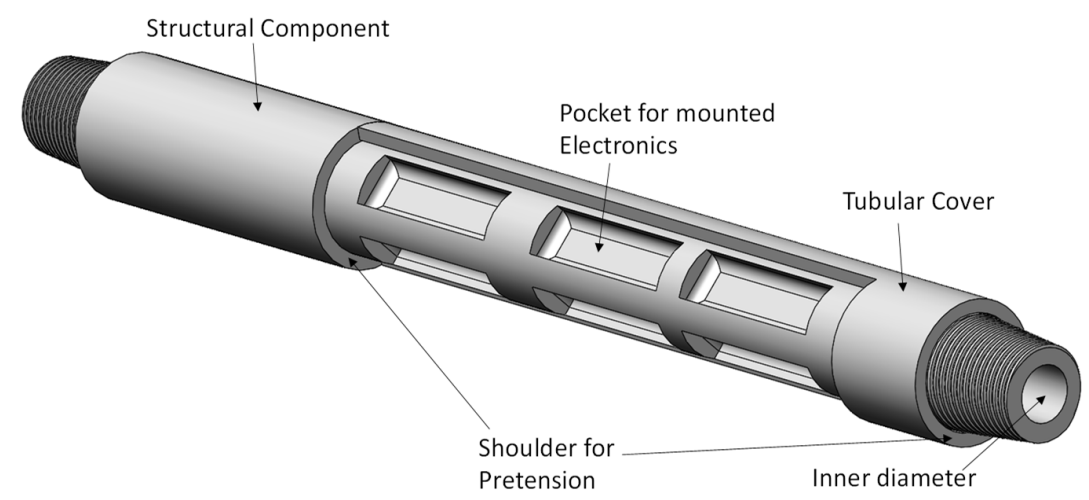

Figure 5. Structural component from the drilling industry

In the following capture step the geometry and topology again investigates the most important and leading parameters. This investigation leads to parameters such as the outside diameter, inside diameter, number of pockets on the circumference, the dimension of the pockets or the radii. In addition, according to the function of the component and the significance of individual parameters, the most important physical domains for the design and scaling process are determined. These include the previously mentioned domains of mechanics, thermodynamics and fluid mechanics. While simultaneously examining the guideline, the most important steps of the design process arise from this. As an extra help previous developments accomplished in the company, as well as the help of experts are used for a more detailed description.

Next, the solution space of the parameters is limited. By using the previously mentioned sizing increments and other specifications concerning adjacent components it is possible to set a variance for each parameter. This action results in a first constraint network that ensures the stability of the model. The component then is divided into single areas, and each area and its individual complexity is documented in the "Entity" group. In connection with the external conditions actual areas with an increased complexity are found. By taking advantage of an iterative loop to the identify step, it is possible to affiliate in what resolution the affected areas should be considered. These areas include for example the influence of the feature shaping at the end of the pocket, resulting in a notch, as well as the stress rise due to the hydrostatic pressure within the inner diameter applied to the structure by the fluid flow in the centre bore. The remaining areas then are classified as analytically predictable.

In the following "Activity" group the guideline previously treated in the "Identify" step is taken into account and by using the already known domains it is possible to create a flow chart that guarantees an adequate design and calculation of the component. Additionally, trade-offs are detected in the mechanical and thermo-dynamical field. The geometry of the pockets influences the mechanical stress by varying the effective moment of inertia. At the same time, the heat development initiated by the mounted electronics and the necessary heat conduction is controlled by the depth of the pocket. Deeper pockets therefore lead to a reduction of the moment of inertia and an increase of the heat conduction. While using the analytical equations it is possible to determine a parameter influence and sensitivity via a dimensionless analysis. Through the formation of a mechanical and thermal dimensionless key figure, the relative influence of the parameters on the respective domains are obtainable. This analysis is then incorporated into the building process of the KBE system and is used as a support in the designing and calculation of the component.

For the areas with increased complexity determined due to the entity points, the parameters for a FEM simulation are set. Due to the fixed increment jumps, the outer diameter is to be modified only in this magnitude. For each increment jump, the pocket depth is varied with fixed inner diameter. Thereupon, the inner diameter is altered by one size and again, the pocket depth is varied.

This is repeated until all sizes are processed and the results are documented in the form of stress values at defined locations in matrix form.

The next step consists in the formalization of these results. For a subsequent application, the approximation of the results and thus the adaption of these results as a case basis to the particular problem are of great significance. For this purpose, the relations of parameter changes are being used, which allow a description of the stress gradient when entering multiple parameters. In combination with the analytically calculated values, it is possible to determine factors that permit an approximation of the stress values. For each 
variation of the inner and outer diameter a new function is derived, so that the whole solution space is describable. Due to the retrievable results for all possible parameter combinations in the solution space related to the case bases together with the analytical calculations and the established scheme, the entire configurator is implemented as a software solution. Especially in a conceptual phase of the development it is possible to verify the best solution for a given problem in a short period of time by just changing the parameter values. Geometrical changes in a CAD program and ongoing FEM calculations can be implemented with an already established concept and therefore the lead time can be reduced.

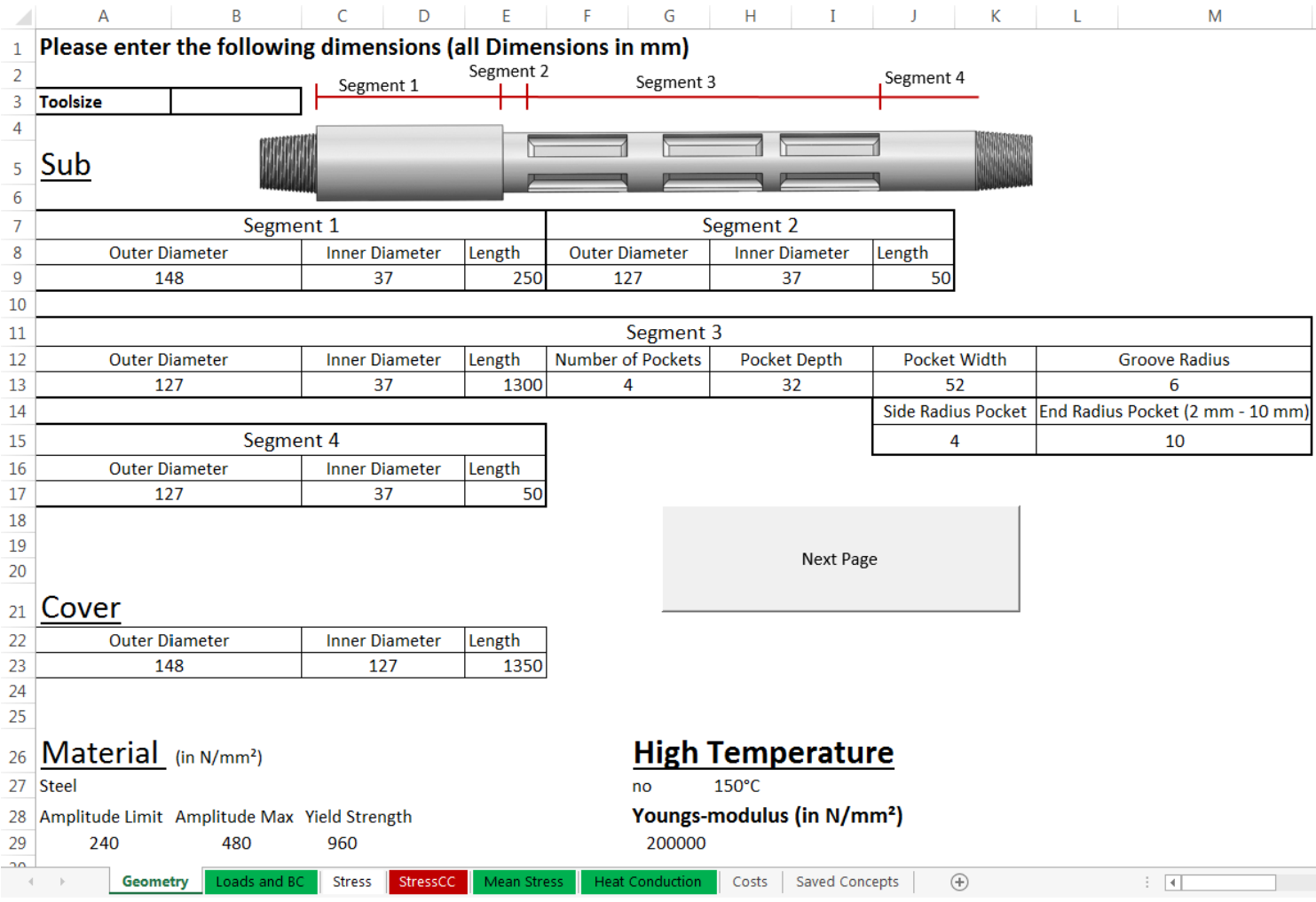

Figure 6. Overview of configurator implemented in Microsoft Excel

Figure 6 gives an overview of the interface of the configurator implemented into the spreadsheet software Microsoft Excel. Several spreadsheets, observable on the bottom of the figure mark the examined domains from the flowchart. With the help of macros it is possible to mark the spreadsheets where required data are not yet inserted or certain boundaries are not fulfilled, what makes the use and the change of geometry parameters with its effect on the outcome easy and quickly accessible. Further Spreadsheets with the calculation formulas as well as the data bases are inserted and cross-referenced to the matching cells.

While using the configurator many outcomes and therefore variants of the component itself are possible. At this stage the user as a subject matter expert has to assess which variant suits his use case the best. While the possibility of a quick change of parameters makes this possible in a short period of time an automatic optimization lead by additional constraints inserted by the user is a further desired research topic. Especially regarding possible trade-offs a self-optimizing geometry would spare several iterations for solving the best outcome of the trade-off.

\section{Conclusion}

This paper proposes a method for the build-up of a KBE system for the scaling of structural components. Based on the Methodology and tools Oriented to Knowledge-based engineering Applications (MOKA), a defined flowchart is derivable for the analysis of the scalable component, the collection of necessary knowledge, the formalization of the knowledge and the later use. The advantage of this method is the defined outcome as a configuration tool for the component scaling with the flexibility of an individual 
adaption of the geometry and of given design features. This leads to a decrease of the development time, especially in the conceptual phase. Steps involving the mechanical simulation with a frequent change of the geometry can be reduced by a quick estimation using the configuration tool from the proposed method. The applicability of the method is shown based on a case study performed on a structural component in the context of the oil and gas industry. But other use cases, beside the oil and gas drilling industry, can benefit in a similar manner from this approach, because of the broad application of structural components in technological products. Topics for a future research are, for example, deriving the approach for complete assemblies with regard to the interfaces of the individual components.

\section{References}

Baker, W.E., Westine, P.S. and Dodge, F.T. (1991), Similarity Methods in Engineering Dynamics: Theory and Practice of Scale Modeling, Elsevier, Amsterdam.

Bridgman, P.W. (1932), Theorie der physikalischen Dimensionen, Teubner, Leipzig.

Deimel, M. (2007), Ähnlichkeitskennzahlen zur systematischen Synthese, Beurteilung und Optimierung von Konstruktionslösungen, $\mathrm{PhD}$ thesis, Technische Universität Braunschweig.

Deimel, M. and Franke, H.-J. (2006), “Ähnlichkeitskennzahlen als Produktdarstellende Modelle zur methodischen Unterstützung der Synthese, Beurteilung und Optimierung von Lösungen”, 4. Gemeinsames Kolloquium Konstruktionstechnik, Kühlungsborn, Germany, September 28-29, 2006, Shaker, Aachen, pp. 149 - 176.

Dutson, A.J. and Wood, K.L. (2002), Foundations and Application of the Empirical Similitude Method (ESM). [online] ResearchGate. Available at: https://www.researchgate.net/publication/270158686_FOUNDATIONS_AND_APPLICATIONS_OF_THE_ EMPIRICAL SIMILITUDE METHOD ESM

Görtler, H. (1975), Dimensionsanalyse - Theorie der physikalischen Dimensionen mit Anwendung, SpringerVerlag, Berlin Heidelberg.

Kline, S.J. (1965), Similitude and Approximate Theory, McGraw-Hill, New York.

Koller, R. (1991), "CAD- und Expertensysteme der Konstruktion - Stand und Möglichkeiten", Berechnung, Gestaltung und Fertigung von Schweisskonstruktionen im Zeitalter der Expertensysteme (EXPERT '91), Essen, Germany, February 20-21, 1991, DVS-Verlag, Düsseldorf, pp. 1-3.

Koschorrek, R. (2007), Systematisches Konzipieren mittels Ähnlichkeitsmethoden am Beispiel von PKWKarosserien, $\mathrm{PhD}$ thesis, Technische Universität Braunschweig.

LaRocca, G. (2012), "Knowledge based engineering: Between AI and CAD - Review of a language based technology to support engineering design”, Advanced Engineering Informatics, Vol. 26 No. 2, pp. 159-179. https://doi.org/10.1016/j.aei.2012.02.002

Lyons, W. and Plisga, G. (2005), Standard Handbook of Petroleum and Natural Gas Engineering, Elsevier Amsterdam.

Moog, W. (1985), Ähnlichkeits- und Analogielehre, VDI-Verlag, Düsseldorf.

Pahl, G., Beitz, W., Feldhusen, J. and Grote, K.-H. (2007), Engineering Design: A Systematic Approach, Springer Science \& Business Media, Berlin Heidelberg. https://doi.org/10.1007/978-1-84628-319-2

Roth, K-H. (2000), Konstruieren mit Konstruktionskatalogen, Springer, Berlin. https://doi.org/10.1007/978-3642-17466-7

Rudolph, S. (2002), Übertragung von Ähnlichkeitsbegriffen, Habilitation thesis, Universität Stuttgart.

Saitou, K., Kazuhiro, I., Nishiwaki, S. and Papalambros, P. (2005), "A survey of Structural Optimization in Mechanical Product Development”, Journal of Computing and Information Science in Engineering, Vol. 5 No. 3, pp. 214-226. https://doi.org/10.1115/1.2013290

Stichlmair, J. (1990), Kennzahlen und Ähnlichkeitsgesetze im Ingenieurwesen, Altos-Verlag, Essen.

Stokes, M. (2001), Managing Engineering Knowledge: MOKA Methodology for knowledge Based Engineering Applications, ASME Press, New York.

Philipp Wolniak, M. Sc.

Leibniz Universität Hannover, Institut für Produktentwicklung und Gerätebau (Institute of product development) Hurlebuschweg 8, 30453 Hannover, Germany

Email:wolniak@ipeg.uni-hannover.de 